\title{
Controversy
}

\section{The analysis by Manuel and colleagues creates controversy with headlines, not data}

\author{
Jacques Genest, Ruth McPherson, Jiri Frohlich, George Fodor
}

ß See related articles pages 1027 and 1037

T

he last 15 years have seen extraordinary changes in the approaches to the prevention and treatment of coronary artery disease (CAD). The high burden and cost of atherosclerotic disease renders its prevention and early detection and treatment extremely important. ${ }^{1,2}$ The publication of the US National Cholesterol Education Program (NCEP) Adult Treatment Panel I report ${ }^{3}$ in 1988 provided a landmark set of recommendations for the detection, evaluation and treatment of high blood cholesterol levels in adults. Subsequent refinements to these recommendations have been prompted and made on the basis of evolving epidemiologic data, findings from clinical studies and advances in drug therapies. ${ }^{4}$ The revised 2003 recommendations for the management of dyslipidemia from the Working Group on Hypercholesterolemia and Other Dyslipidemias are an example of this evolution.

Guidelines must adapt to advances in medicine and be periodically reviewed. They and their authors should be open for debate and criticism. Debates can be catalysts for change, much like catalysts of chemical reactions. Like many chemical reactions, some debates create more heat than light; the commentary by Manuel and colleagues in this issue of $C M A 7$ (page 1027) 6 is, alas, one such example.

Controversy is not new to the debate about cholesterol and $\mathrm{CAD}$. More than a decade ago, on the basis of the data then available, Ravnskov concluded that "lowering serum cholesterol concentrations does not reduce mortality and is unlikely to prevent coronary artery disease." More recently, the Therapeutics Initiative group from British Columbia asserted that "statins have not been shown to provide an overall health benefit in primary prevention trials." ${ }^{8}$ Under pressure from some members of the medical community, a follow-up Therapeutics Initiative letter conceded that lowering cholesterol levels with statins is beneficial in the secondary prevention of CAD. ' Today's evidence tells us more. The authors of the recent INTERHEART study, the largest case-control study to date, involving 15152 patients with myocardial infarction and 14820 control subjects from 52 countries, estimated the population attributable risk of myocardial infarction due to abnormal lipid levels (i.e., apolipoprotein B/AI, a surrogate for the total cholesterol:high-density lipoprotein cholesterol ratio) to be $49.2 \% .^{10}$ The study demonstrates that abnormal lipid levels, along with smoking status, hypertension, obesity, diet, alcohol, physical activity and psychosocial factors, account for most of the risk of myocardial infarction worldwide in both sexes and at all ages in all regions. ${ }^{11}$

The INTERHEART study reinforces the central theme in our revised recommendations, seemingly missed by Manuel and colleagues, that clinicians should take a risk factor approach and give priority treatment to patients at high risk of a first or recurrent cardiac event as determined by history of disease or the risk calculation algorithm that is based on the Framingham study. This preventive strategy follows principles first used in New Zealand and then adopted by the NCEP III ${ }^{11}$ and the 2003 Canadian guidelines. ${ }^{5}$ A targeted population-based strategy is recommended, based on age, the presence of risk factors and the calculation of a 10-year risk of CAD-related death or a nonfatal myocardial infarction. A risk of $20 \%$ or greater is arbitrarily defined as high, between $10 \%$ and $19 \%$ as moderate and less than $10 \%$ as low. The lipid level targets for the 3 risk categories are more stringent the higher the risk. A target low-density lipoprotein cholesterol level less than $5.0 \mathrm{mmol} / \mathrm{L}$ is suggested for patients at very low risk. The main reason for this is to give clinicians room to use their judgement not to treat relative extremes of low-density lipoprotein cholesterol levels while not depriving patients with severe hypercholesterolemia of treatment. Familial hypercholesterolemia rarely makes it on the radar screen of epidemiologists, yet in the province of Quebec alone it is estimated that about 30000 people have the condition.

The main point of contention in the analysis by Manuel and colleagues centres on the number of people at low risk who might be prescribed statins according to our revised 2003 recommendations. Their analysis is based on 2 items: a reference population obtained from the Canadian Heart Health Survey (CHHS) and extrapolations from the LaRosa meta-analysis ${ }^{12}$ for assessing the benefit of treatment. There are several flaws in their analysis, including the lipid target value selected for patients at low risk, the quality and currency of the reference population data, the appropriateness of extrapolations about the effectiveness of lipid therapy made from the results of the LaRosa metaanalysis, and omissions of certain key populations at risk.

Manuel and colleagues used a low-density lipoprotein cholesterol cutoff point of $4.5 \mathrm{mmol} / \mathrm{L}$ for low-risk subjects. We clearly stated that a cutoff point of $5.0 \mathrm{mmol} / \mathrm{L}$ would be acceptable in subjects with a very low 10 -year risk. Their analysis does not reflect this important point. 
The reference CHHS population sample is based on about 18000 subjects who had a blood sample taken between 1988 and 1992. Although a good reference population and perhaps the best data available to Manuel and colleagues, the CHHS dataset has serious limitations. It is outdated and underestimates the current proportions of Canadians at low, medium and high risk of heart disease. Over the past 15 years, there has been a steady increase in the number of Canadians with obesity and other components of the metabolic syndrome, a newly recognized constellation of endocrine disturbances that doubles the risk of cardiovascular events. The proportion of Canadians at cardiovascular risk is therefore higher in $2003^{3,13}$ and underestimated by use of the CHHS sample. Moreover, contrary to the statement and implications made by Manuel and colleagues, the 2003 Canadian guidelines do not recommend widespread screening in men younger than 40 years or in premenopausal women.

Manuel and colleagues estimate the magnitude of the benefit of statin therapy in terms of deaths prevented by maintaining therapy for high-risk patients whose lipid levels are acceptably low on the basis of extrapolations from the LaRosa meta-analysis. Their rationale for choosing this particular source is not clear. There are several studies from which to infer and extrapolate data. Much more appropriate, recent and relevant to the Canadian context would have been the Heart Protection Study ${ }^{14}$ which used a more potent statin (simvastatin $40 \mathrm{mg}$ ) than some of the studies in the metaanalysis and demonstrated a greater magnitude of effect.

In addition to concerns about the appropriateness of the CHHS and LaRosa sources, we question the way Manuel and colleagues present their results. Since estimates of effects vary, it is customary to present results within a statistical range say, $95 \%$ confidence intervals. This was not done here. Applying these statistical considerations would considerably weaken the authors' assertions that hundreds of deaths and millions of dollars would be saved if the 2003 guidelines were revised.

Finally, the authors used self-reported disease status for diabetes. This seems unjustified and undoubtedly resulted in an underestimation of the proportion of respondents with diabetes. Surely the CHHS must have had data on serum glucose levels. If not, it only reinforces our concern about the quality and appropriateness of this database. Moreover, the analysis overlooks the importance of genetics in overall risk. The 2003 Canadian guidelines list family history of premature $\mathrm{CAD}$ as a factor that nearly doubles the 10 -year risk. Data on family history were not available in the CHHS.

The analysis by Manuel and colleagues highlights some of the challenges in designing methodologies to establish and stratify cardiovascular risk in subjects with few risk factors. These subjects make up the majority of the population at risk of cardiovascular disease and in need of primary prevention. Methodology should not override the importance of sound clinical judgement. Statin therapy should not be initiated for the sole purpose of lowering an elevated cholesterol level; however, most candidates for therapy also have 1 or more risk factors.
Manuel and colleagues would have been more persuasive had they been more candid in admitting the limitations of their analysis. We maintain that cardiovascular risk stratification and targeted therapy is a cost-effective strategy for disease prevention, and we have not seen data to convince us otherwise. In our estimate Manuel and colleagues have produced a little heat, but not much light.

From the Division of Cardiology, Royal Victoria Hospital, McGill University, Montréal, Que. (Genest), the University of Ottawa Heart Institute, Ottawa, Ont. (McPherson, Fodor) and St. Paul's Hospital, University of British Columbia, Vancouver, BC (Frohlich)

Competing interests: Jacques Genest is a paid consultant for and has received speaker fees from Merck Frosst, Schering-Plough, Pfizer Canada and SanofiAventis; he has received travel assistance from Bayer. Phyllis McPherson has received speaker fees from various pharmaceutical firms, including Pfizer Canada, Merck Frosst/Schering Pharmaceuticals and AstraZeneca. Jiri Frohlich has received speaker fees and travel assistance from producers of lipid-lowering medications, including Pfizer Canada, Merck Frosst, AstraZeneca and Fournier Pharma. George Fodor has received a research and an educational grant from Merck Frosst and Pfizer Canada as well as speaker fees from Merck Frosst and AstraZeneca.

\section{References}

1. Yusuf S, Reddy S, Ounpuu S, Anand S. Global burden of cardiovascular diseases: part I: general considerations, the epidemiologic transition, risk factors, and impact of urbanization.Circulation 2001;104:2746-53.

2. Yusuf S, Reddy S, Ounpuu S, Anand S. Global burden of cardiovascular diseases: Part II: variations in cardiovascular disease by specific ethnic groups and geographic regions and prevention strategies. Circulation 2001;104:2855-64.

3. Expert Panel on Detection, Evaluation, and Treatment of High Blood Cholesterol in Adults. Executive summary of the third report of the National Cholesterol Education Program (NCEP) Expert Panel on Detection, Evaluation and Treatment of High Blood Cholesterol in Adults (Adult Treatment Panel III). 7AMA 2001;285:2486-97.

4. Genest J, Petersen TR. Prevention of cardiovascular ischemic events: high risk and secondary prevention. Circulation 2003;107:2059-65.

5. Genest J, Frohlich J, Fodor G, McPherson R (the Working Group on Hypercholesterolemia and Other Dyslipidemias). Recommendations for the management of dyslipidemia and the prevention of cardiovascular disease: summary of the 2003 update. CMA7 2003;169(9):921-4.

6. Manuel DG, Tanuseputro P, Mustard CA, Schultz SE, Anderson GM, Ardal $\mathrm{S}$, et al. The 2003 Canadian recommendations for dyslipidemia management: Revisions are needed. CMA7 2005;172(8):1027-31.

7. Ravnskov U. Cholesterol lowering trials in coronary artery disease: frequency of citation and outcome. BMF 1992;350:15-9.

8. Therapeutics Initiative. Do statins have a role in primary prevention? Ther Lett 2003;48 (April-June). Available: www.ti.ubc.ca/pages/letter48.htm (accessed 2005 Mar 10)

9. Therapeutics Initiative. Statin's benefit for secondary prevention confirmed. What is the optimal dosing strategy? Ther Lett 2003;49 (July-August). Available: www.ti.ubc.ca/pages/letter49.htm (accessed 2005 Mar 10).

10. Yusuf S, Hawken S, Ounpuu S, Dans T, Azevum A, Lanas F, et al, on behalf of the INTERHEART study investigators. Effect of potentially modifiable risk factors associated with myocardial infarction in 52 countries (the INTERHEART study): case-control study. Lancet 2004;364:937-52.

11. Grundy SM, Cleeman JI, Merz CN, Brewer HB Jr, Clark LT, Hunninghake DB, et al; National Heart, Lung, and Blood Institute; American College of Cardiology Foundation; American Heart Association. Implications of recent clinical trials for the National Cholesterol Education Program Adult Treatment Panel III guidelines. Circulation 2004;110:227-39.

12. LaRosa JC, He J, Vupputuri S. Effect of statins on risk of coronary disease: a meta-analysis of randomized controlled trials. 7AMA 1999;282:2340-6.

13. Katzmarzyk PT, Ardern CI. Overweight and obesity mortality trends in Canada, 1985-2000. Can 7 Public Health 2004;95:16-20.

14. Heart Protection Study Collaborative Group. MRC/BHF Heart Protection Study of cholesterol lowering with simvaststin in 20536 high-risk individuals: a randomized placebo-controlled trial. Lancet 2002;360:7-22.

Correspondence to: Dr. Jacques Genest, Division of Cardiology, Royal Victoria Hospital, 687 Pine Ave. W., Montréal QC H3A 1A1; fax 514 843-2813; jacques.genest@muhc.mcgill.ca 